\title{
Motion Analysis and Segmentation for Object- Oriented Mid-Level Image Representation
}

\author{
Takahiro Saito and Takashi Komatsu \\ Department of Electrical Engineering, Kanagawa University \\ Yokohama, 221, Japan
}

\begin{abstract}
In the object-oriented mid-level image representation, moving images are decomposed into multiple global image regions undergoing different coherent motion, and then each global coherent motion region is described with its texture and region map along with its motion model. With regard to the global motion segmentation, this paper employs the strategy of a gradual migration from a simple local optical flow representation of motion to a compound global segmentation representation of motion, and presents a new matching-based robust local motion estimation algorithm with which we design to cope with the generalized aperture problem. Furthermore, this describes an iterative global motion segmentation algorithm for decomposing moving images into multiple global coherent motion regions, each of which conforms to a different global motion model.
\end{abstract}

\section{Introduction}

Image coding and computer vision are closely related domains, and we can apply a hierarchical framework of computer vision, ranging from low-level image representation to high-level image representation, to image coding. Low-level image coding have been well studied so far, but it is said that low-level image coding is now approaching the performance limit. On the other hand, the high-level concept is now considered to be beyond reach except in restricted domains [1].

Object-oriented mid-level image representation is considered to be a promising candidate for the image representation with which a fertile domain of new-generation generic image coding will be opened up. Recently, many research groups are studying mid-level image coding [2, 3]. Mid-level image representations involve such concepts and attributes as surfaces, segmentation, global motion, texture, transparency, and so on. Wang and Adelson have recently advocated a layered image representation [3]. Their layered representation is to describe moving images with sets of overlapping layers, each of which contains three different maps: the intensity map, the alpha map, and the velocity map. The layers are ordered in depth and occlude each other in accord with the rule of composition. They have presented some methods for decomposing image sequences into layers using a global motion segmentation algorithm, and they have employed the standard strategy of a gradual migration from a local optical flow representation of motion to a global segmentation representation of motion. Local motion estimation and motion segmentation are decisively important for generating stable accurate mid-level image representation. An inaccurate local optical flow representation at the first stage of the global motion segmentation will greatly damage quality of the resultant global motion segmentation.

Our work keeps within the framework of mid-level image representation and employs the standard strategy of the gradual migration. In the first stage of the gradual 
migration strategy, local optical flow estimation is carried out over a local neighborhood, and hence the generalized aperture problem, which means the dilemma surrounding the proper size of an analysis image block, appears to be the most serious problem with local motion estimation. To solve the problem, this paper extends the basic concept of the existing block-matching algorithm, thus forming a matchingbased robust local motion estimation algorithm based on the assumption that there may be multiple distinct image patterns undergoing different coherent motion within a given analysis image block. Furthermore, this paper presents an iterative global motion segmentation algorithm which identifies multiple global coherent motion regions by initially fitting global perspective motion models to local optical flow estimates recovered by the extended matching-based robust local motion estimation algorithm and then refining segmentation of multiple global coherent motion regions and their corresponding global perspective motion models alternately iteratively.

\section{Extended Matching-Based Algorithm for Estimating Multiple Local Image Motions}

We employ the standard strategy of the gradual migration from a simple local optical flow representation of motion to a compound global segmentation representation of motion. In the local optical flow representation of motion, we assume that each local image pattern within an analysis image block undergoes a simple rigid translation, while in the global segmentation representation of motion we employ a perspective transformation as a motion model. The gradual migration strategy circumvents the egg-and-chicken dilemma surrounding interdependence between motion analysis and segmentation, and alleviates the difficulty of recovering compound motion model parameters directly from a given input image sequence. This chapter concentrates on a new-extended matching-based algorithm for recovering the local optical flow representation of motion from a given input image sequence.

\subsection{Generalized Aperture Problem in Local Motion Analysis}

In local motion estimation, the size of the analysis image block is a critical factor. A large analysis image block is needed to sufficiently constrain the solution and to provide some insensitivity to noise. The larger the analysis image block is made, the less likely the assumption about the motion will be valid over the entire analysis image block. For example, the constant velocity assumption will be violated by an affine and or perspective optical flow, motion boundaries, transparency, and so on. The dilemma surrounding the proper size of an analysis image block is referred to as the generalized aperture problem.

The appropriate size of an analysis image block depends on such factors as the size and the velocity of objects in the scene, and hence it is extremely difficult to determine the optimal size of an analysis image block. Therefore, there may be a number of local motion configurations occurring within an analysis image block when its size is arbitrary determined $[4,5,6]$.

The existing local motion analysis methods can deal with only the simplest local motion configuration where the block contains a single image pattern undergoing coherent motion. This paper presents a new robust matching-based local motion estimation algorithm which can deal with the more complex local motion configuration where the block consists of multiple distinct image patterns undergoing coherent motion. 
Recently, the study group of Bergen and others, and the study group of Shizawa and others, have independently presented the extended gradient-based algorithms which can deal with the other complex local motion configuration of multiple transparent image patterns $[4,5]$. Their algorithms are constructed with the second derivatives of image intensity, and hence unfortunately seems to be sensitive to noise. Our algorithm is robust even under real noisy conditions like the existing block matching algorithm, but it requires large computational efforts.

\subsection{Prototypal Two-Frame Algorithm [6]}

For the analysis of multiple motion vectors, firstly the extended algorithm segments a given analysis image block into multiple distinct image regions undergoing coherent motion, simultaneously applies its matching mechanism to each image region separately, and then provides its multiple matching results as multiple motion vector estimates; the extended algorithm finally provides a single motion estimate for each image region. A segmentation process is usually based on image intensity, but the segmentation process introduced here is based on the assumption that each image region undergoes coherent motion.

We form the extended two-frame algorithm as the following discrete optimization problem, where $\mathrm{p}$ motion vectors $\left(\mathrm{m}_{1}, \mathrm{n}_{1}\right), \ldots \ldots,\left(\mathrm{m}_{\mathrm{p}}, \mathrm{n}_{\mathrm{p}}\right)$ are determined so that the cost function is minimized.

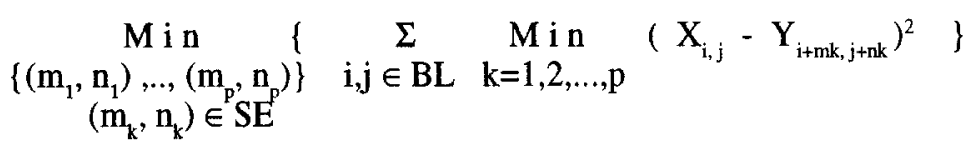

$X_{i, j}$ : image intensity of the pixel $(i, j)$ in the present image frame $X$

$Y_{i, j}^{i, j}$ : image intensity of the pixel $(i, j)$ in the next image frame $Y$

$\mathrm{SE}$ : search area, BL : analysis image block

In addition, we have experimentally derived a good way of determining the proper value of the number of image regions $p$. The way is as follows: we start with the initial value of 1 for $p$, and then we increase $p$ by one if and only if the increase of $p$ by one decreases the minimum cost value to less than $20 \%$ of the preceding minimum cost value before the increase of $p$.

\subsection{Improved Algorithms}

Three-Frame Algorithm. Generally speaking, the two frame algorithm has a major drawback. The two-frame algorithm cannot handle the image areas covered by other moving image patterns, and it cannot provide correct motion vector estimates in the covered areas, because the covered area does not appear in the next image frame. To solve this problem, we should introduce the concept of a three-frame algorithm which estimates multiple motion vectors by applying the extended segmental matching process to consecutive three image frames. We form the three-frame algorithm as the following discrete optimization problem, where $p$ motion vector estimates $\left(m_{1}\right.$, $\left.\mathrm{n}_{1}\right), \ldots \ldots,\left(\mathrm{m}_{\mathrm{p}}, \mathrm{n}_{\mathrm{p}}\right)$ are determined so that the cost function is minimized. 


$$
\begin{aligned}
& \left.\underset{\left\{\left(m_{1}, n_{1}\right), \ldots,\left(m_{p}, n_{p}\right)\right\}}{M} \underset{i, j \in B L}{\sum} \underset{k=1,2, \ldots, p}{M i n}\left\{\left(X_{i, j}-Y_{i-m k, j-n k}\right)^{2},\left(X_{i, j}-Z_{i+m k, j+n k}\right)^{2}\right\}\right\} \\
& \left(\mathrm{m}_{\mathrm{k}}, \mathrm{n}_{\mathrm{k}}\right) \in \mathrm{SE} \\
& X_{i, j} \text { : image intensity of the pixel }(i, j) \text { in the previous image frame } X \\
& Y_{i, j} \text { : image intensity of the pixel }(i, j) \text { in the present image frame } Y \\
& Z_{i, j} \text { : image intensity of the pixel }(i, j) \text { in the next image frame } Z \\
& \mathrm{SE} \text { : search area, BL : analysis image block }
\end{aligned}
$$

Introduction of Spatial Coherence Constraint. The prototypal extended algorithm has another defect that it does not take the spatial coherence property of a motion field into consideration. The spatial coherence property means the assumption that surfaces have spatial extent and hence neighboring pixels with similar pixel intensity value in an image are likely to belong to the same surface and likely to have similar motion vectors.

By imposing the spatial coherence constraint to a motion vector estimate assigned to each pixel within an analysis image block, the extended algorithm will probably get to provide correct motion vector estimates even for a pixel in the vicinity of which there are few salient features. The spatial coherence constraint is introduced into the extended three-frame algorithm as follows; firstly we segment an analysis image block into small cells by grouping neighboring pixels having similar pixel intensity into a small cell, and then in the local motion estimation procedure expressed by equation (2) we select an optimal motion vector estimate for each segmented small cell, instead of for each pixel, from among $p$ motion vector candidates, on the assumption that pixels which belong to the same segmented small cell have the same motion vector. The algorithm is organized as follows:

[Spatial Coherence Constraint Algorithm]

(1) If the difference between the maximum pixel intensity value and the minimum pixel intensity value within an analysis image block, typically with $16 \times 16$ pixels, exceeds a threshold $\mathrm{Th}$, then we divide an analysis image block into four small square cells equally.

(2) If the condition applied in the step (1) is satisfied in a segmented small cell, then we divide the segmented small cell further into four smaller cells equally. This procedure is repeated until the size of the segmented small cell reaches the prescribed smallest cell size, typically of $2 \times 2$ pixels, or further segmentation is not performed.

(3) We solve the optimization problem of equation (2) under the constraint that pixels which belong to the same segmented small cell should be allotted the same motion vector estimate.

\subsection{Evaluation}

As an instance, this paper presents only the results of the experiment using the natural image sequence where the doll is swinging like a pendulum, but in this image sequence the doll looks as if it were undergoing pure horizontal translation, and the vertical component of the optical flow field is nearly zero for every pixel in the image. In the experimental simulations, the size of an analysis image block and that of a search area are fixed at $16 \times 16$ and $13 \times 13$ respectively.

Fig. 1 shows the results of the extended matching-based three-frame algorithm with the spatial coherence constraint. The left column shows the horizontal components of 
the motion estimates and the right column shows the vertical components of the motion estimates. In Fig. 1, pixel intensity is allocated to each pixel according to its estimated motion component. The existing block-matching algorithm incurs estimation errors along the contour of the moving doll. The prototypal extended twoframe algorithm decreases estimation errors along the contour of the moving doll to some extent, but the estimated flow field is rather rugged along the contour. On the other hand, the extended three-frame algorithm with the spatial coherence constraint decreases estimation errors along the moving doll considerably, and recovers the flow field as accurately as we expect.

\section{Iterative Global Motion Segmentation}

The iterative global motion segmentation algorithm identifies multiple global image regions, each of which undergoes a different coherent motion, by fitting perspective motion models to local optical flow estimates recovered with the local motion estimation algorithm described in the previous chapter.

The iterative global motion segmentation algorithm is organized as follows:

(1) Initial Perspective Motion Model: Beginning with the local optical flow representation of motion, we determine a initial set of global coherent motion regions that have spatially continuous optical fields, by grouping neighboring pixels having spatially continuous optical flows into a global coherent motion region, and then we determine an initial set of perspective motion models each of which well approximates the optical flow field within each global coherent motion region with the standard weighted least square regression algorithm. The perspective motion model is defined by the equations

$$
\begin{aligned}
& v_{x}(x, y)=a+b x+c y+d x^{2}+e x y \\
& v_{y}(x, y)=f+g x+h y+e y^{2}+d x y
\end{aligned}
$$

where $v_{x}$ and $v_{y}$ are the $x$ and $y$ components of velocity, $(x, y)$ are the spatial coordinates, and (a,b,c,d,e,f,g,h) are the parameters of the model.

(2) Iterative Refinement of Motion Segmentation: At each iteration step, multiple

horizontal component $\mathrm{u}$

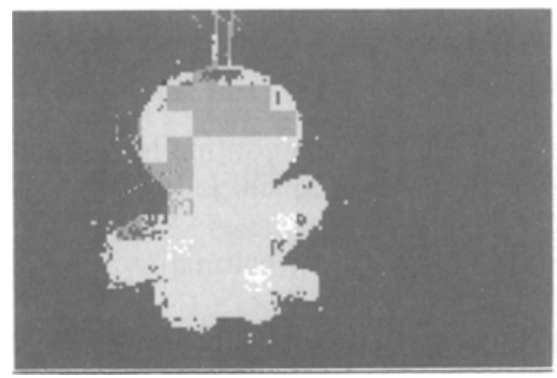

vertical component $\mathrm{v}$

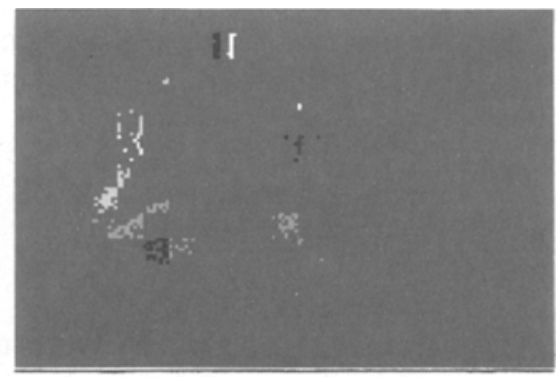

Fig. 1. Motion vector estimates provided by the extended three-frame algorithm with the spatial coherence constraint. 
perspective motion models and multiple global motion regions are alternately refined until convergence. At each iteration step, segmentation of multiple global coherent motion regions is updated not only by comparing the local optical flow estimates with the multiple perspective motion models, but also by estimating intensity differences between the observed image frame and its corresponding image frame warped with each of the multiple perspective motion models, and then a proper perspective motion model is newly calculated within each of the refined global coherent motion regions by the standard gradient-based weighted least square motion estimation algorithm based on the perspective motion model.

(3) Interframe Projection of Motion Segmentation (Temporal Integration): The perspective motion models and the segmentation of successive image frames will be similar, because a rigid object shape and motion change slowly from frame to frame. To preserve temporal coherency and continuity, we use the current global motion segmentation results as initial segmentation for the next image frame. Once the global motion segmentation is completed on the entire image sequence, each global coherent motion region will be identified and tracked in the entire image sequence with its corresponding perspective motion model and its spatial location and extent.

We have conducted experiments using the identical natural image sequence to evaluate the performance of the entire process of the iterative global motion segmentation algorithm. The iterative global motion segmentation algorithm works very well for the natural test image sequence.

\section{Conclusion}

We present a new matching-based robust local motion estimation algorithm and an iterative global motion segmentation algorithm, which are characterized as key technologies for recovering a mid-level image representation from a given input image sequence. We have conducted experiments using natural image sequences to evaluate the performance of the algorithms. The results of the experiments demonstrate that for natural image sequences the algorithms work as well as we expect.

\section{References}

1.K. Aizawa, H. Harashima, T. Saito: Model-based analysis synthesis image coding system for person's face, Signal Processing: Image Communication 1, 139152(1989)

2.M. Hotter, R. Thoma: Image segmentation based on object oriented mapping parameter estimation, Signal Processing 15, 315-334(1988)

3.J.W.A Wang, E.H. Adelson: Representing moving images with layers, IEEE Trans. Image Processing 3, 625-638(1994)

4.B. Bergen, P.J. Burt, R. Hingonari, S. Peleg; A three-frame algorithm for estimating two-component image motion, IEEE Trans. Patt. Anal. Machine Intell. 14, 886896(1992)

5.M. Shizawa, K. Mase: Simultaneous multiple optical flow estimation, Proc. Int. Conf. Patt. Recog., 274-278(1990)

6.T. Komatsu, T. Saito: Extended block-matching algorithm for estimation of multiple image motion, The Journal of the Institute of Television Engineers of Japan 49, 328335(1995) 